\title{
Factors associated with development of gastrointestinal problems in patients with scleroderma: a protocol for a systematic review
}

Brian Younho Hong ${ }^{1 *}$, Raymond Giang ${ }^{2}$, Lawrence Mbuagbaw ${ }^{3,4,5}$, Maggie Larche ${ }^{6}$ and Lehana Thabane $3,4,7,8,9$

\begin{abstract}
Background: Scleroderma affects the gastrointestinal (GI) tract in $90 \%$ of all cases. Malnutrition, diarrhea, and constipation are some $\mathrm{Gl}$ complications that can stem from scleroderma, and they contribute considerably to impairment in quality of life. Reports of haphazard clusters of high prevalence suggest that environmental exposure is a risk factor for scleroderma. However, it is largely uncertain whether the Gl involvement secondary to scleroderma is influenced by these environmental factors. This study will review the association between $\mathrm{Gl}$ involvement (unintentional weight loss, choking, early satiety, etc.) and environmental exposure in patients with scleroderma.

Methods/design: Any available observational studies that report GI problems in patients with scleroderma along with the associated risk factors will be selected. We will search CINAHL, EMBASE, LILACS, MEDLINE, and Web of Science for relevant articles written in English from June 1884 to May 2014. Identified articles will be screened in duplicate, and full text for selected articles will be retrieved. Data extraction will be done in duplicate on sociodemographic characteristics of participants, diagnosis of scleroderma, diagnosis of risk of Gl problem, risk factors reported, etc. Discrepancies will be resolved by consensus or by consulting a third author. We will assess the participants, methods, and intervention effects of included studies for heterogeneity. Any identified clinical or statistical heterogeneity will be explored visually or using the chi-square test. Data will be pooled statistically using the DerSimmonian and Laird random effects method if we have a measure of relative risk and its precision. Our findings will be reported according to the Meta-Analyses and Systematic Review of Observational Studies (MOOSE) guideline.
\end{abstract}

Discussion: Our findings may help patients with scleroderma and health care professionals in preventing Gl morbidity. Knowing that the cost of care for patients with scleroderma increases with more organ involvement, study findings can inform policy developers to identify ways to curb health care costs.

Systematic review registration: PROSPERO: CRD42014010707

Keywords: Systematic review, Scleroderma, Gastrointestinal, Risk factor

\footnotetext{
* Correspondence: brian.youn.hong@learnlink.mcmaster.ca

${ }^{1}$ Bachelor of Health Sciences (Honours) Program, McMaster University,

Hamilton, ON, Canada

Full list of author information is available at the end of the article
} 


\section{Background}

Scleroderma, otherwise known as systemic sclerosis, is a disease manifested by collagen overproduction affecting various organs including the gastrointestinal (GI) tract [1]. Patients with scleroderma commonly present with inflammation and fibrosis of the skin, vascular abnormalities, organ damage, and autoantibody production [2]. Diagnosis is made when these signs and corresponding symptoms are present [3]. Scleroderma is a debilitating disease that is estimated to affect approximately 16,000 Canadians, impacting women four to five times more so than men [1]. The prevalence of the disease is higher among those of African origin and varies across countries, appearing higher in North America and Australia than in Europe and Japan [4]. Scleroderma is associated with debilitating morbidity such as reduction in mobility and depression [5]. Patients with this disorder have $30.8 \%$ mortality rate, although this number can vary depending on gender and what visceral organs are involved [6]. Currently, there are no effective treatments to combat scleroderma, partly because its pathogenesis remains unclear [7]. Consequently, much attention has shifted towards predicting complications secondary to scleroderma and to manage them appropriately [8]. The underlying rationale is that it is easier to treat the stage before these complications arise [8].

The GI tract issues are involved in 90\% of all scleroderma patients and contribute considerably to impairment in quality of life [9-11]. Malnutrition, nausea, vomiting, diarrhea, and constipation are some GI complications that can stem from scleroderma [9]. Reports of nonlinear clusters of high prevalence suggest that environmental exposure is a risk factor for scleroderma [11]. For example, silica along with silicone and epoxy resins have been associated with increased risk of scleroderma [11]. It is largely uncertain, however, whether the GI involvement secondary to scleroderma is influenced by these environmental factors. This study will review the association between GI involvement and environmental exposure in patients with scleroderma. These findings will help patients with scleroderma and health care professionals in preventing the GI morbidity. In Canada, the average annual economic burden of scleroderma is estimated to be $\$ 18,453$ per patient [12]. Knowing that the cost to care for patients with scleroderma increases with more organ involvement, the study findings can inform policy developers to identify ways to curb health care costs [12].

The overall purpose of this systematic review is to determine the factors associated with development of GI problems in patients with scleroderma. We will look for risk factors for GI problems in patients with scleroderma. These risk factors include silica, silicone implantation and rupture, antacid, solvents, epoxy resins, welding fumes, anorexigens, pentazocine, bromocriptine, L-tryptophan, pesticides, constant hand/arm vibration, and alcohol [13-18]. We will consider additional risk factors, such as intestinal microbiota and food, in the event that we find any studies that report on them. We will identify and report other predictors of and factors associated with development of GI problems in scleroderma patients.

\section{Methods/design \\ Inclusion/exclusion criteria \\ Types of studies}

Any available observational studies that report on GI problems in patients with scleroderma along with the associated risk factors will be selected for the review.

They must have the following criteria:

1. Patients with a formal diagnosis of scleroderma according to 1980 and 2013 criteria for the diagnosis of systemic sclerosis by the American College of Rheumatology $[19,20]$.

2. Clearly reported GI problems.

3. Specific risk factors for GI problems will be studied.

\section{Study settings}

Literature from any geographic or socioeconomic setting will be used.

\section{Types of participants}

All participants will be included provided they have scleroderma and are assessed for risk factors for GI problems.

\section{Intervention/exposure}

We will look for risk factors for GI problems in patients with scleroderma. These risk factors may include silica, silicone implantation and rupture, antacid, solvents, epoxy resins, welding fumes, anorexigens, pentazocine, bromocriptine, L-tryptophan, pesticides, constant hand/arm vibration, and alcohol [13-18]. We will consider additional risk factors, such as intestinal microbiota and food, in the event that we find any studies that report on them. We will identify and report other predictors of and factors associated with development of GI problems in scleroderma patients.

\section{Search strategy for identification of studies}

We shall attempt to identify all studies relevant to the GI problems in patients with scleroderma written in English from June 1884 to May 2014. June 1884 was chosen because it is the earliest entry of scleroderma in available databases.

\section{Electronic searches}

We will search Cumulative Index to Nursing and Allied Health Literature (CINAHL), Excerpta Medica database (EMBASE), Latin American and Caribbean Health Sciences Literature (LILACS,) Medical Literature Analysis and 
Retrieval System Online (MEDLINE), and Web of Science for relevant articles written in English from June 1884 to April 2014. We will use the subject term services of the different databases. The following search terms and their medical subject heading ( $\mathrm{MeSH}$ ) equivalents will be used: scleroderma, risk factors, gastrointestinal, unintentional weight loss, poor appetite, dysphagia, acid reflux, choking, early satiety, bloating, nausea, vomiting, constipation, diarrhea, antibiotic use, steatorrhea, fecal incontinence, parenteral nutrition, and the above listed risk factors. These terms will be used in varying combinations. Table 1 is a proposed search strategy for MEDLINE via Ovid.

\section{Reference lists}

We will manually check the reference list of all the studies identified by the above search strategy. Relevant studies shall be read and included in the review when deemed appropriate. We will also check the reference list of any previous reviews on GI problems secondary to scleroderma for any relevant articles, which could be included in the review.

\section{Gray literature}

We will attempt to contact authors, experts in the field, research organizations, and conference websites for any relevant material.

\section{Data collection and analysis Screening}

Two authors (BYH and RJ), working independently, will screen all citations and abstracts resulting from the search strategy to identify eligible papers. The full text of eligible articles will be obtained and assessed using a pre-designed eligibility form based on the inclusion criteria. Eligible studies will be included in the review. Disagreements will be resolved by discussion or by seeking the opinion of a third party (LT/ML).

Authors will be contacted to clarify missing or unclear information. Ineligible studies will be excluded from the review, and the reason for exclusion will be stated in a table.

\section{Data extraction}

The two authors (BYH and RJ) will independently extract data from the studies using a pre-established data extraction form. Data will be extracted on sociodemographic characteristics of participants (age, gender, occupation, residence), diagnosis of scleroderma, diagnosis of risk of GI problem, risk factors reported, study design, study setting, sample size, lost to follow-up/missing data, and strength of association between risk factors and outcome, if reported.

In order to pool the risk factors for GI problems among the participants with scleroderma, we will extract the numbers with each identified risk factor among those
Table 1 Proposed search strategy of ovid

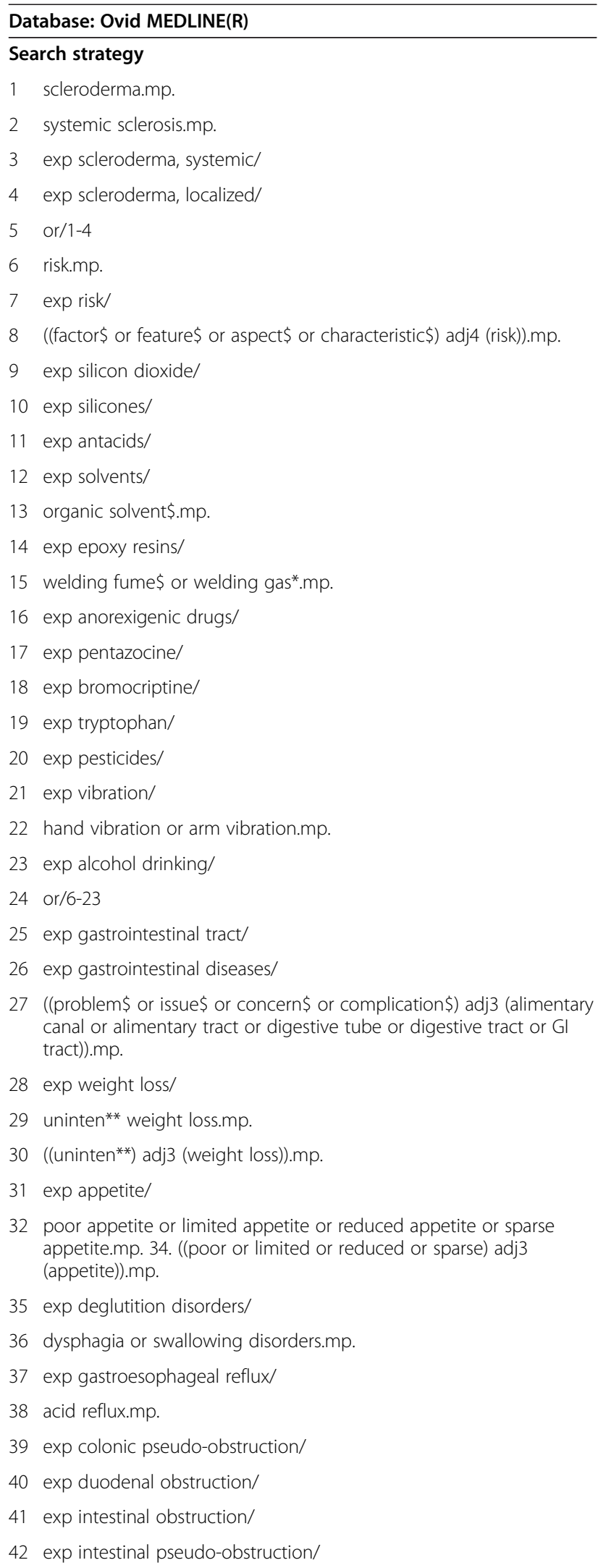




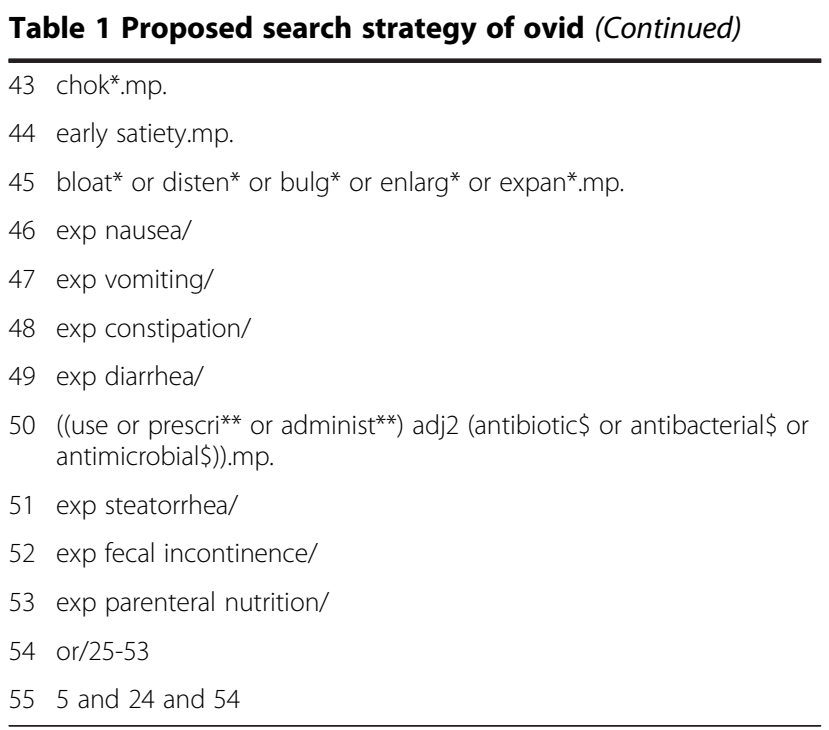

with GI problems and the numbers without the risk factor so we can construct two-by-two tables and use them for meta-analyses. If only the odds ratios are reported with a measure of precision (confidence interval or standard error), we will use this in the meta-analysis.

\section{Assessment of methodological quality}

The two authors (BYH and RJ) will independently assess the methodological quality of included studies using the Newcastle-Ottawa Scale [21]. We will be assessing publication bias using funnel plots along with other biases such as reporting bias, performance bias, and detection bias.

\section{Analyses and reporting}

\section{Data synthesis and assessment/investigation of heterogeneity}

We will only pool the results if there is a measure of relative risk (risk ratio, odds ratio, hazard ratio) and its precision (95\% confidence intervals, standard error, variance). We will assess the participants, methods, and intervention effects of included studies for heterogeneity. This will enable us to determine whether results can be pooled across studies. Any identified clinical or statistical heterogeneity will be explored visually or using the chi-square test. Possible sources of heterogeneity include the population studied, the severity of the condition, the severity of the exposure, duration of follow-up, etc.

\section{Statistical analysis}

Comparison of risk will be made against scleroderma patients with the risk of GI concerns who have not developed GI problems. Data will be pooled statistically using the DerSimmonian and Laird random effects method where appropriate [22]. The results will be expressed as odds ratios with 95\% confidence interval and $p$ values.
Between-study heterogeneity will be tested using Cochran's $\mathrm{Q}$ and the $I^{2}$ statistic. Stratified analysis of children versus adults and inpatients versus outpatients may be conducted. Data will be analyzed using Review Manager version 5.2.

\section{Sensitivity analyses}

A sensitivity analysis will be performed to explore how robust the results are to variations in methodological quality.

\section{Presenting and reporting of results}

The analysis and reporting of the review will be done according to the Meta-Analyses and Systematic Review of Observational Studies (MOOSE) guideline [23]. We will use a flow diagram to summarize the selection process of the studies. Agreement between data abstractors will be assessed using the kappa statistic [24]. A full list of excluded studies with reasons for exclusion will be reported.

\section{Discussion}

GI manifestations secondary to scleroderma are multifaceted depending on the parts of the alimentary tract involved [11]. Severe GI problems are associated with higher rates of mortality [25]. Studies report that $90 \%$ of scleroderma patients experience GI problems underscoring the quality of life concerns associated with them [11]. The information obtained from the study will be important for patients, clinicians, and policy developers. By identifying and reporting predictors of and factors associated with GI problems in scleroderma patients along with early biomarkers that can predict the future severity of the disease, this study will help clinicians to improve risk assessment. The resultant earlier and more effective diagnosis can aid patients in reducing the GI morbidity. By identifying ways to prevent GI morbidity, this study can curb the cost of health care in helping scleroderma patients.

\section{Ethics and dissemination}

The results of this paper will be disseminated as peerreviewed publications, at conferences and at clinical rounds.

\section{Abbreviations \\ CINAHL: Cumulative Index to Nursing and Allied Health Literature; EMBASE: Excerpta Medica database; Gl: gastrointestinal; LILACS: Latin American and Caribbean Health Sciences Literature; MEDLINE: Medical Literature Analysis and Retrieval System Online; MOOSE: Meta-Analyses of Observational Studies in Epidemiology. \\ Competing interests \\ The authors declare that they have no competing interests.}

\section{Authors' contributions}

$B Y H, M L$, and $L T$ conceived of the study, revised the research question, and provided content to the design. RG and LM revised the research question and provided content to the design. All authors read and approved the final version of the manuscript. 


\section{Author details}

'Bachelor of Health Sciences (Honours) Program, McMaster University, Hamilton, ON, Canada. ${ }^{2}$ Statistics for Health (Honours), University of Waterloo, Waterloo, ON, Canada. ${ }^{3}$ Department of Clinical Epidemiology and Biostatistics, McMaster University, Hamilton, ON, Canada. ${ }^{4}$ Biostatistics Unit, Father Sean O'Sullivan Research Centre, St. Joseph's Healthcare, Hamilton, ON, Canada. ${ }^{5}$ Centre for Development of Best Practices in Health, Yaoundé Central Hospital, Yaoundé, Cameroon. ${ }^{6}$ Division of Rheumatology, Departments of Medicine and Paediatrics, McMaster University, St. Joseph's Healthcare, McMaster Children's Hospital, Hamilton, ON, Canada.

${ }^{7}$ Departments of Paediatrics and Anaesthesia, McMaster University, Hamilton, ON, Canada. ${ }^{8}$ Centre for Evaluation of Medicine, St. Joseph's Healthcare, Hamilton, ON, Canada. ${ }^{9}$ Population Health Research Institute, Hamilton Health Sciences, Hamilton, ON, Canada.

Received: 6 August 2014 Accepted: 3 October 2014

Published: 13 October 2014

\section{References}

1. Canadian Scleroderma Research Group. http://canadian-sclerodermaresearch-group.ca/.

2. Al-Dhaher FF, Pope JE, Ouimet JM: Determinants of morbidity and mortality of systemic sclerosis in Canada. Semin Arthritis Rheum 2010, 39(4):269-277.

3. Hachulla E, Launay D: Diagnosis and classification of systemic sclerosis. Clin Rev Allergy Immunol 2011, 40(2):78-83.

4. Ranque B, Mouthon L: Geoepidemiology of systemic sclerosis. Autoimmun Rev 2010, 9(5):A311-A318.

5. Malcarne VL, Hansdottir I, McKinney A, Upchurch R, Greenbergs HL, Henstorf GH, Furst DE, Clements PJ, Weisman MH: Medical signs and symptoms associated with disability, pain, and psychosocial adjustment in systemic sclerosis. J Rheumatol 2007, 34(2):359-367.

6. Ferri C, Valentini G, Cozzi F, Sebastiani M, Michelassi C, La Montagna G, Bullo A, Cazzato M, Tirri E, Storino F, Giuggioli D, Cuomo G, Rosada M, Bombardieri S, Todesco S, Tirri G, Systemic Sclerosis Study Group of the Italian Society of Rheumatology: Systemic sclerosis: demographic, clinical, and serologic features and survival in 1,012 Italian patients. Medicine 2002, 81(2):139-153.

7. Smith V, Riccieri V, Pizzorni C, Decuman S, Deschepper E, Bonroy C, Sulli A, Piette Y, De Keyser F, Cutolo M: Nailfold capillaroscopy for prediction of novel future severe organ involvement in systemic sclerosis. I Rheumatol 2014, 40(12):2023-2028.

8. Smith V, Decuman S, Sulli A, Bonroy C, Piettte Y, Deschepper E, de Keyser F, Cutolo M: Do worsening scleroderma capillaroscopic patterns predict future severe organ involvement? A pilot study. Ann Rheum Dis 2012, 71(10):1636-1639.

9. Baron M, Bernier P, Cote F, DeLegge MH, Falovitch G, Friedman G, Gornitsky M, Hoffer L, Hudson M, Khanna D, Paterson WG, Schafer D, Toskes PP, Wykes $L$ : Screening and management of malnutrition and related gastro-intestinal disorders in systemic sclerosis: recommendations of a North American expert panel. Clin Exp Rheumatol 2010, 28(Suppl. 58):S42-S46.

10. Khanna D, Nagaraja V, Gladue H, Chey W, Pimentel M, Frech T: Measuring response in the gastrointestinal tract in systemic sclerosis. Curr Opin Rheumatol 2013, 25(6):700-706.

11. Chifflot H, Fautrel B, Sordet C, Chatelus E, Sibilia J: Incidence and prevalence of systemic sclerosis: a systematic literature review. Semin Arthritis Rheum 2008, 37(4):223-235.

12. Bernatsky S, Hudson M, Panopalis P, Clarke AE, Pope J, Leclerca S, St Pierre Y, Baron M, Canadian Scleroderma Research Group: The cost of systemic sclerosis. Arthritis Care Res 2009, 61(1):119-123.

13. Agarwal SK, Tan FK, Arnett FC: Genetics and genomic studies in scleroderma (systemic sclerosis). Rheum Dis Clin North Am 2008, 34:17-40.

14. Lidar M, Agmon-Levin N, Langevitz P, Shoenfeld Y: Silicone and scleroderma revisited. Lupus 2012, 21(2):121-127.

15. Wielosz E, Borys O, Zychowska I, Majdan M: Gastrointestinal involvement in patients with systemic sclerosis. Pol Arch Inter Med 2010, 120(4):132-136.

16. Diot E, Lesire V, Guilmot JL, Metzger MD, Pilore R, Rogier S, Stadler M, Diot $P$, Lemarie $E$, Lasfargues $G$ : Systemic sclerosis and occupational risk factors: a case-control study. Occup Environ Med 2002, 59(8):545-549.
17. Marie I, Gehanno JF, Bubenheim M, Duval-Modeste AB, Joly P, Dominique S, Bravard P, Noël D, Cailleux AF, Weber J, Lagoutte P, Benichou J, Levesque H: Prospective study to evaluate the association between systemic sclerosis and occupational exposure and review of the literature. Autoimmun Rev 2014, 13(2):151-156.

18. Silman AJ: Epidemiology of scleroderma. Ann Rheum Dis 1991, 50:846-853.

19. Subcommittee for scleroderma criteria of the American Rheumatism Association Diagnostic and Therapeutic Criteria Committee: Preliminary criteria for the classification of systemic sclerosis (scleroderma). Arthritis Rheum 1980, 23(5):581-590.

20. van den Hoogen F, Khanna D, Fransen J, Johnson SR, Baron M, Tyndall A, Matucci-Cerinic M, Naden RP, Medsger TA Jr, Carreira PE, Riemekasten G, Clements PJ, Denton CP, Distler O, Allanore Y, Furst DE, Gabrielli A, Mayes MD, van Laar JM, Seibold JR, Czirjak L, Steen VD, Inanc M, Kowal-Bielecka O Muller-Ladner U, Valentini G, Veale DJ, Vonk MC, Walker UA, Chung L, et al: 2013 classification criteria for systemic sclerosis. Ann Rheum Dis 2013, 72(11):1747-1755.

21. Stang A: Critical evaluation of the Newcastle-Ottawa scale for the assessment of the quality of nonrandomized studies in meta-analyses. Eur J Epidemiol 2010, 25(9):603-605

22. Deeks JJ, Altman DG, Bradburn MJ: Statistical methods for examining heterogeneity and combining results from several studies in meta-analysis. In Systematic Reviews in Health Care. 2nd edition. Edited by Egger M, Smith GD, Altman DG. London: BMJ Publishing; 2001:285-312.

23. Stroup DF, Berlin JA, Morton SC, Olkin I, Williamson GD, Rennie D, Moher D, Becker BJ, Sipe TA, Thacker SB: Meta-analysis of observational studies in epidemiology: a proposal for reporting. Meta-analysis of Observational Studies in Epidemiology (MOOSE) group. JAMA 2000, 283:2008-2012

24. McGinn T, Wyer PC, Newman TB, Keitz S, Leipzig R, For GG, Evidence-Based Medicine Teaching Tips Working G: Tips for learners of evidence-based medicine: 3. Measures of observer variability (kappa statistic). Can Med Assoc J 2004, 171(11):1369-1373.

25. Steen VD, Medsger TA: Severe organ involvement in systemic sclerosis with diffuse scleroderma. Arthritis Rheum 2000, 43(11):2437-2444.

doi:10.1186/2046-4053-3-115

Cite this article as: Hong et al.: Factors associated with development of gastrointestinal problems in patients with scleroderma: a protocol for a systematic review. Systematic Reviews 2014 3:115.

\section{Submit your next manuscript to BioMed Central and take full advantage of:}

- Convenient online submission

- Thorough peer review

- No space constraints or color figure charges

- Immediate publication on acceptance

- Inclusion in PubMed, CAS, Scopus and Google Scholar

- Research which is freely available for redistribution 